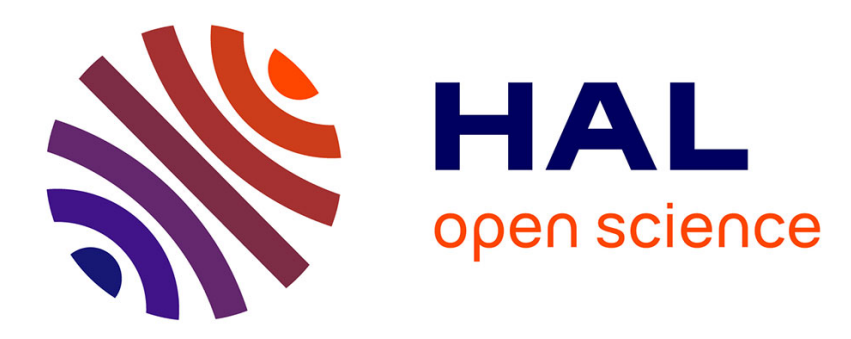

\title{
Bistabilité optique des lasers couplés
}

\author{
J.L. Oudar, R. Kuszelewicz
}

\section{To cite this version:}

J.L. Oudar, R. Kuszelewicz. Bistabilité optique des lasers couplés. Revue de Physique Appliquée, 1987, 22 (10), pp.1287-1295. 10.1051/rphysap:0198700220100128700 . jpa-00245679

\section{HAL Id: jpa-00245679 https://hal.science/jpa-00245679}

Submitted on 1 Jan 1987

HAL is a multi-disciplinary open access archive for the deposit and dissemination of scientific research documents, whether they are published or not. The documents may come from teaching and research institutions in France or abroad, or from public or private research centers.
L'archive ouverte pluridisciplinaire HAL, est destinée au dépôt et à la diffusion de documents scientifiques de niveau recherche, publiés ou non, émanant des établissements d'enseignement et de recherche français ou étrangers, des laboratoires publics ou privés. 
Classification

Physics Abstracts

42.60D, F - 42.55P $-42.80 \mathrm{~S}$

\title{
Bistabilité optique des lasers couplés
}

\author{
J. L. Oudar et R. Kuszelewicz \\ Centre National d'Etudes des Télécommunications, 196, avenue Henri-Ravera, 92220 Bagneux, France
}

(Reçu le 4 décembre 1986, accepté le 12 février 1987)

\begin{abstract}
Résumé. - On écrit le principe et les méthodes de réalisation d'une classe de bistable optique à basculement symétrique, basée sur l'inhibition réciproque de deux lasers à grand gain. L'analyse théorique permet de décrire les conditions d'obtention du régime bistable et les possibilités de commutation par voie purement optique. Une expérience menée avec une paire de lasers à colorant confirme la validité de ce principe et démontre la possibilité de commutations sub-nanoseconde. L'extension aux lasers à semi-conducteurs est discutée et les caractéristiques fonctionnelles de ces dispositifs sont comparées à celles des autres bistables optiques.
\end{abstract}

\begin{abstract}
We describe the principle and several possible implementations of an optical flip-flop, based on the mutual quenching of coupled high-gain lasers. Our theoretical analysis describes the requirements for bistable operation and the conditions of purely optical switching. Experiments with a dye laser pair confirm the validity of this approach and demonstrate the possibility of subnanosecond switching. Extension to semiconductor lasers is discussed and the functional features of these devices are compared to those of other optical bistable devices.
\end{abstract}

\section{Introduction.}

Depuis quelques années, certains efforts de recherche se sont concentrés sur la possibilité d'utiliser les phénomènes non linéaires optiques pour réaliser diverses fonctions de la logique booléenne sur des faisceaux lumineux [1]. Ceux-ci pouvant être facilement codés pour véhiculer des signaux, on peut ainsi envisager un traitement tout optique de l'information, qui bénéficierait d'un certain nombre d'avantages sur le plan de la bande passante, et du parallélisme, rendu possible par la maîtrise des techniques d'imagerie. Les dispositifs de traitement tout optique doivent permettre de traiter et transformer les signaux optiques sans nécessiter comme c'est le cas actuellement les conversions optoélectroniques et électro-optiques. Dans le domaine des télécommunications par fibres optiques, ces conversions sont très fréquentes, et il est clair qu'il y aurait avantage à simplifier cette procédure. La reconfiguration des interconnexions est également un problème majeur dans les circuits de traitement de l'information, que ce soit à l'intérieur d'un ordinateur ou d'un central de télécommunications. Là, généralement, des solutions purement optiques sont possibles par le biais des connexions optiques reprogrammables [2]. Les dispositifs de traitement tout optique apparaissent donc, à côté des composants électroniques et optoélectroniques, comme la probable troisième génération de composants des technologies de l'information.

Les systèmes présentant une caractéristique de bistable optique sont tout indiqués pour réaliser des fonctions logiques optiques [3]. En effet ils possèdent par définition deux états, et l'on peut en général faire passer le système d'un état à l'autre par le biais d'une commande optique. De plus, il suffit en général de modifier légèrement certains paramètres de ces systèmes pour obtenir des caractéristiques de transfert sans hystérésis mais néanmoins fortement non linéaires. Ceci permet, par l'existence d'un seuil de basculement du système, soit les remises en forme nécessaires aux traitements en cascade, soit les opérations logiques à plusieurs entrées.

Parmi les bistables optiques on distingue ceux qui sont par eux-mêmes émetteurs de lumière, et ceux qui agissent par modification des caractéristiques d'un faisceau lumineux incident, au cours de la transmission ou de la réflexion de ce faisceau. Ces deux catégories de bistables ont chacune leurs avan- 
tages et leurs inconvénients. Les bistables émissifs sont plus attrayants pour la mise en cascade de plusieurs traitements, car dans leur principe même ils sont capables d'émettre, même en l'absence de faisceau de commande. Par contre les bistables transmissifs présentent souvent l'avantage d'une plus grande simplicité de structure (en particulier les bistables intrinsèques), qui permet d'envisager plus commodément des configurations matricielles pour le traitement des points d'une image bidimensionnelle. Ces distinctions ne sont toutefois pas à prendre au sens strict, car les mises en cascade sont malgré tout possibles avec les bistables transmissifs, et d'autre part on peut très bien concevoir, avec les lasers à semi-conducteurs émettant perpendiculairement à la surface, des configurations bidimensionnelles de bistables émissifs. Ces distinctions apparaissent donc surtout lorsque l'on compare les facilités de mise en œuvre pour tel ou tel type d'application.

Dans la suite de cet article nous décrirons quelques résultats obtenus sur un type particulier de bistable émissif : celui résultant du couplage en intensité de deux lasers à grand gain [4]. Le principe de fonctionnement de ce type de bistable est décrit dans la section 2, ainsi que les différentes configurations envisageables. Dans la section 3 seront décrits quelques résultats expérimentaux et théoriques obtenus dans notre laboratoire sur ce type de bistable. Enfin dans la section 4 seront discutés les aspects fonctionnels qui leur sont propres, en particulier ceux dus à la nature symétrique (ou complémentaire) du mode de fonctionnement.

\section{Bistabilité résultant du couplage non cohérent de deux lasers.}

Considérons tout d'abord l'action d'un faisceau extérieur sur un laser, comme représenté sur la figure 1. Nous supposons que l'inversion de population de celui-ci le maintient au-dessus du seuil en l'absence du faisceau extérieur. Si le faisceau extérieur a une longueur d'onde suffisamment proche de

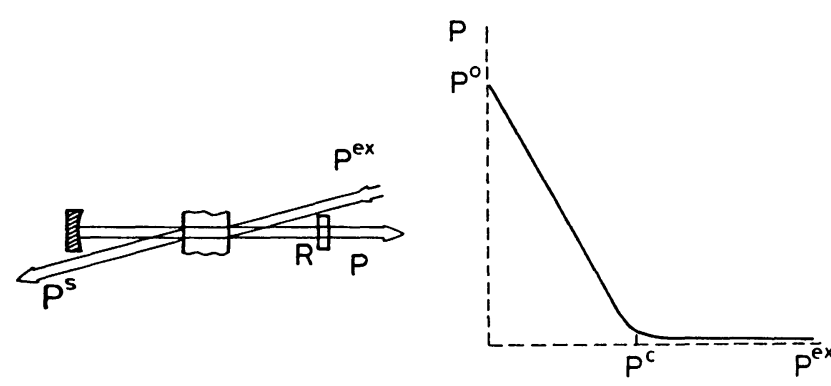

Fig. 1. - Action d'un faisceau externe sur l'émission d'un laser. L'augmentation progressive de la puissance injectée conduit, pour une puissance critique $P^{\mathrm{c}}$ à l'extinction de l'émission laser.

[Action of an external beam on the laser emission. The laser emission can be extinguished for a critical value $P^{c}$ of the injected beam power.] celle émise normalement par le laser, pour être amplifiée par le milieu actif, cette amplification se fait au détriment de la puissance émise par le laser. En conséquence (voir Fig. 1), si l'on augmente progressivement la puissance $P^{\mathrm{ex}}$ du faisceau extérieur, la puissance $P$ du laser doit passer progressivement de la valeur nominale $P^{\circ}$, lorsque $P^{\mathrm{ex}}=0$, à une valeur nulle, lorsque $P^{\text {ex }}$ dépasse une valeur critique $P^{\mathrm{c}}$. On voit là un exemple de la possibilité de contrôler l'intensité d'un faisceau lumineux, au moyen d'une commande purement optique. On note que ce contrôle est de type inhibiteur, c'est-à-dire que la puissance émise par le laser diminue lorsque la puissance de contrôle augmente. On note également que l'action inhibitrice est d'autant plus grande que la valeur $P^{\mathrm{c}}$ nécessaire pour éteindre le laser est faible.

On peut alors concevoir un montage comportant deux lasers similaires, arrangés de telle sorte que la sortie d'un des lasers constitue le faisceau de contrôle de l'autre, et réciproquement. On obtient alors une configuration à inhibition réciproque, telle que, si certaines conditions sont réunies, l'émission d'un des lasers bloque l'émission de l'autre, ou vice versa. On peut prévoir deux états possibles pour le système : 1) le laser 1 émet et bloque le laser 2 et 2) le laser 2 émet et bloque le laser 1 . Pour examiner plus en détail les conditions dans lesquelles cela peut se produire, il convient de considérer les diagrammes de la figure 2 , où l'un des axes représente la puis-

(a)
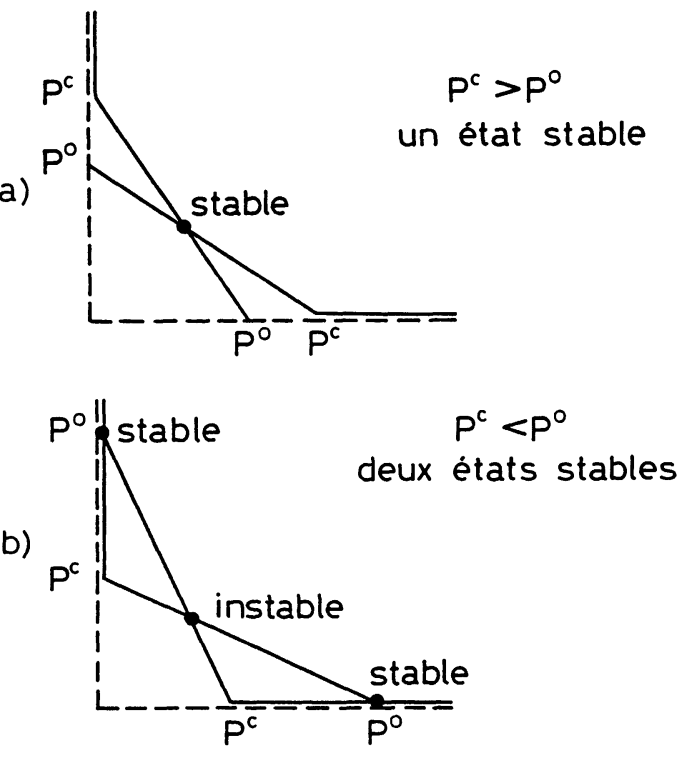

Fig. 2. - Etats de fonctionnement de deux lasers mutuellement inhibés. L'apparition de deux régimes : (a) non bistable et (b) bistable réside dans la valeur relative de la puissance de sortie $P^{\circ}$ et de la puissance critique $P^{\mathrm{c}}$.

[Operating points of mutually quenched twin-lasers. Two different regimés can be observed : (a) stable ; (b) bistable, depending on the relative values of the output power $P^{\circ}$ and the critical power $P^{\mathrm{c}}$.] 
sance de sortie du laser 1, et l'autre la puissance de sortie du laser 2. Pour simplifier on suppose que la puissance de sortie du laser 2 est utilisée entièrement pour commander le laser 1 , et réciproquement. Sur ces diagrammes, on a donc à représenter la superposition de deux courbes du même type que sur la figure 1, disposées symétriquement par rapport à la bissectrice des deux axes, si les deux lasers sont de caractéristiques identiques. Les états d'équilibre du système correspondent aux points d'intersection de ces deux courbes. Deux cas de figure sont alors possibles, selon la valeur relative de $P^{\mathrm{c}}$ par rapport à $P^{\circ}$ : si $P^{\mathrm{c}}>P^{\circ}$ les courbes ne se croisent qu'en un point, correspondant à un état du système où les deux lasers émettent une puissance égale, mais ne se perturbent pas suffisamment pour que l'émission de l'un exclue l'émission de l'autre. Par contre, si $P^{\mathrm{c}}<P^{\circ}$ les courbes se croisent en trois points : en plus de l'état symétrique situé sur la bissectrice existent deux états antisymétriques situés à proximité des axes. Dans chacun de ces deux nouveaux états l'un des lasers est maintenu en dessous du seuil par le faisceau émis par l'autre. On vérifie en considérant de petits écarts par rapport à la situation d'équilibre, que lorsqu'un seul point d'intersection existe il correspond à un équilibre stable ; par contre, dans le cas de trois points d'intersection, l'état symétrique est instable, alors que les états antisymétriques sont stables, conformémemt à ce qui était anticipé. On conclut donc dès lors que l'action inhibitrice d'un laser sur l'autre est suffisamment forte pour que $P^{\mathrm{c}}<P^{\circ}$. Des modèles plus ou moins raffinés peuvent

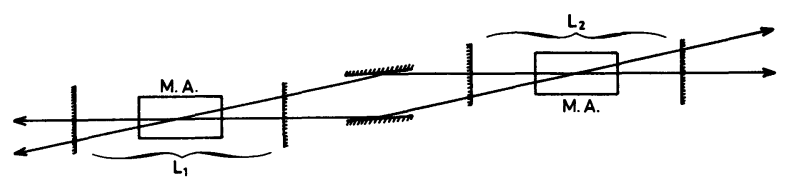

(a)
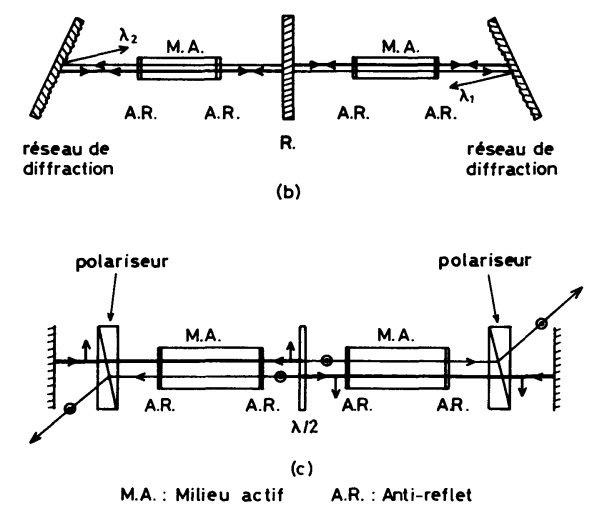

Fig. 3. - Méthodes de discrimination : (a) par la direction de propagation; (b) par la longueur d'onde d'émission ; (c) par la direction de polarisation.

[Means of discrimination : (a) by the propagation direction ; (b) by the emission wavelength ; (c) by the polarization direction.]

REVUE DE PHYSIQUE APPLIQUÉE. - T. 22, N 10, OCTOBRE 1987 être proposés pour évaluer l'intensité de cette action inhibitrice, et nous décrirons un de ces modèles dans la section 3 . Il est néanmoins possible de se contenter du raisonnement suivant [4] :

Dans une courbe comme celle de la figure 1 , lorsque $P^{\mathrm{ex}}<P^{\mathrm{c}}$, le laser est au-dessus du seuil bien que sa puissance soit réduite. Il est bien connu qu'en régime stationnaire la puissance de sortie $P$ du laser est telle que le gain saturé est égal aux pertes. Par conséquent, la puissance extraite du milieu actif par le faisceau extérieur réduit de la même quantité la puissance de sortie $P$ :

$$
P=P^{\circ}-\left(P^{\mathrm{s}}-P^{\mathrm{ex}}\right)
$$

où $P^{\mathrm{s}}$ est la puissance du faisceau externe à la sortie du milieu amplificateur. Pour une puissance d'entrée $P^{\mathrm{ex}}$ donnée, il est clair que la réduction de $P$ est d'autant plus grande que le rapport d'amplification $P^{\mathrm{s}} / P^{\text {ex }}$ est plus important. On se tournera donc vers des milieux amplificateurs à grand gain, tels que les lasers à colorants ou les lasers à semi-conducteurs, et des cavités faiblement résonnantes pour maintenir un gain saturé élevé.

Différentes configurations peuvent être envisagées pour réaliser ce type de bistable optique. Elles diffèrent par la façon dont on réalise l'extraction du faisceau externe hors de la seconde cavité. Celle-ci nécessite un moyen physique pour discriminer les faisceaux externe et interne à une cavité déterminée. La figure 3 représente schématiquement les trois méthodes de discrimination possibles : par la direction de propagation, par la longueur d'onde, et par la polarisation. La première est la plus simple à mettre en œuvre, mais introduit certaines contraintes géométriques qui tiennent à la nécessité de maintenir un volume d'interaction aussi grand que possible entre l'onde externe et l'onde interne dans chaque milieu actif, afin de ne pas diminuer le coefficient de couplage entre ces deux ondes. Lorsque la géométrie $\mathrm{du}$ milieu actif ne permet pas l'injection en biais du faisceau externe (en particulier dans le cas de structures à ondes guidées), les discriminations par la longueur d'onde et par la polarisation restent possibles. Les axes des deux cavités étant identiques, il est possible, et même préférable qu'un miroir soit commun aux deux cavités : de cette façon on réalise un système de deux sous-cavités, qui restent indépendantes l'une de l'autre quant à leurs structures de mode.

La discrimination en longueur d'onde, telle qu'elle est représentée dans la figure 3 , repose sur le fait que chacun des réseaux placés aux extrémités ne réinjecte suivant l'axe de la cavité qu'une longueur d'onde particulière, dépendant de l'orientation du plan du réseau par rapport à l'axe de la cavité (montage Littrow). Si les deux réseaux sont accordés sur deux longueurs d'onde différentes $\lambda_{1}$ et $\lambda_{2}$, 
toutes deux situées dans la région amplificatrice des milieux actifs, chaque sous-cavité constituée du miroir commun, d'un milieu actif et d'un réseau, peut entrer en oscillation-laser indépendamment de l'autre sous-cavité. Comme les longueurs d'onde $\lambda_{1}$ et $\lambda_{2}$ sont différentes, l'émission résonnante d'une sous-cavité n'est pas réinjectée suivant l'axe de la cavité par le réseau fermant l'autre sous-cavité, ce qui réalise la discrimination en longueur d'onde.

De la même manière la discrimination par polarisation se fait en incorporant deux polariseurs entre miroir extérieur et milieu actif de chaque souscavité, et en orientant ces polariseurs de telle sorte que l'émission résonnante d'une sous-cavité soit éjectée par le polariseur de l'autre sous-cavité. Ceci peut se faire, soit en « croisant » les deux polariseurs suivant deux directions orthogonales, soit, comme il est représenté sur la figure 3, en les maintenant parallèles (pour préserver la symétrie du dispositif) mais en remplaçant le miroir de couplage par une lame demi-onde $(\lambda / 2)$ dont les lignes neutres sont à $45^{\circ}$ de celles des polariseurs, et éventuellement munie de traitements diélectriques pour en modifier la réflectivité.

On peut noter qu'en l'absence des discriminateurs en longueur d'onde ou en polarisation, on aurait affaire à des cavités couplées dont la structure de modes longitudinaux dépendrait de manière critique $\mathrm{du}$ rapport des longueurs entre sous-cavités. En particulier ceci est le cas des lasers « $\mathrm{C}^{3}$ " à cavitéscouplées-clivées [5], qui ne sont pas considérés ici, bien qu'ils puissent donner lieu à la bistabilité optique.

\section{Résultats expérimentaux et théoriques.}

Une expérience décrite plus en détail en [4] a été effectuée pour démontrer la faisabilité du principe de bistabilité optique décrit au paragraphe précédent. Des lasers à colorants pompés optiquement ont été utilisés, car ils permettent, au moins en régime de pompage impulsionnel, de réaliser des milieux amplificateurs de grand gain avec des paramètres géométriques qui se prêtent aisément au découplage angulaire décrit sur la figure 3a. Pour que les conditions de bistabilité soient satisfaites en dépit d'un couplage géométrique réduit par l'incidence oblique du faisceau extérieur, les résonateurs sont constitués de deux miroirs à faible réflectivité (Fig. 4) : les parois de verre de la cuve utilisée pour contenir la solution de colorant procurent une réflectivité $R=0.04$ de chaque côté du milieu actif, leur parallélisme étant suffisant pour obtenir l'effet laser après excitation optique du milieu actif. Celui-ci est constitué d'une solution de rhodamine 6G dans l'éthanol, excité transversalement par le second harmonique d'un laser Nd: YAG fonctionnant en régime monomode déclenché. Les impulsions de

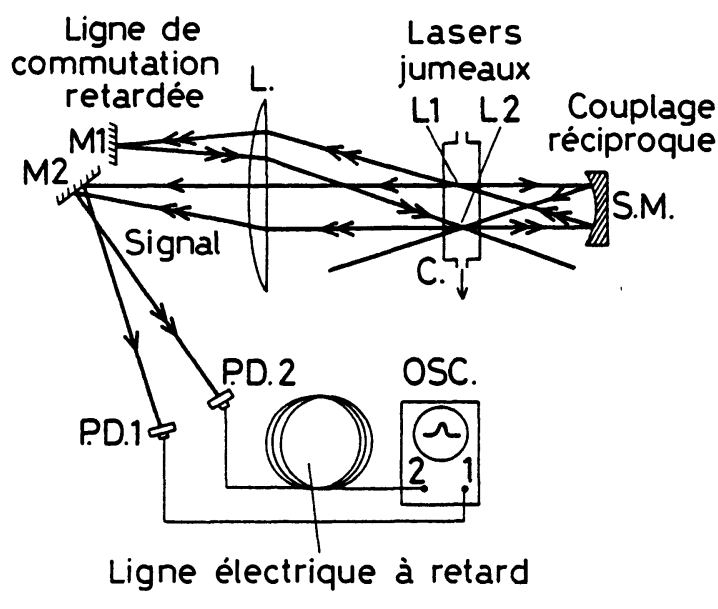

Fig. 4. - Schéma du montage bistable utilisant 2 cavités laser à colorant et une ligne de commutation à retard. M1, M2 : miroirs plans ; L : lentille ; S.M. : Miroir Sphérique ; PD1, PD2 : photodiodes rapides ; $\mathrm{C}$ : cellule à colorant ; osc : oscilloscope.

[Schematic view of the bistable dye cell twin-laser experimental set-up comprising a delayed switching line. M1, M2 : flat mirrors ; L : lens, S.M. : Spherical Mirror ; PD1, PD2 : fast photodiodes ; C : dye cell ; osc : oscilloscope.]

pompe, d'une durée de $20 \mathrm{ns,} \mathrm{sont} \mathrm{suffisamment}$ longues devant la durée vie des photons dans les cavités-lasers (17 ps) et la durée de vie de l'inversion de population (quelques ns) dans le colorant, pour que l'on puisse observer clairement sur un oscillogramme les basculements occasionnés par la bistabilité optique, et les distinguer de l'évolution temporelle des mêmes lasers en régime habituel. Le couplage mutuel entre les deux lasers est assuré par un miroir sphérique, dont le centre de courbure est situé à mi-chemin entre les deux milieux actifs, qui sont juxtaposés dans la même cuve de colorant.

Avec ce dispositif il a été observé en mesurant la puissance d'émission de chaque laser que lorsque le couplage est suffisant, les lasers sont effectivement mis en compétition : en modifiant progressivement le pompage relatif des deux milieux actifs, on passe brutalement d'une situation où l'un des lasers, nommons-le $\mathrm{L} 1$, est presque éteint, à la situation inverse où c'est L2 qui ne peut dépasser le seuil d'oscillations. Ceci indique clairement une situation bistable, mais ne permet pas d'estimer le temps de basculement, car le pompage est impulsionnel et la transition entre les deux états a lieu en quelque sorte dans l'intervalle séparant deux impulsions de pompe. Pour remédier à cet inconvénient, nous nous sommes placés dans des conditions telles que le système puisse basculer pendant une impulsion de pompage. Pour cela, on contrôle le pompage relatif de façon à ce que le laser L2 soit favorisé et inhibe L1; à l'aide de miroirs on renvoie l'émission de L2 sur son propre milieu actif après un certain retard (environ 
$2 \mathrm{~ns})$. Ainsi le front de montée retardé de l'émission de L2 provoque une auto-extinction prématurée de celui-ci. Ceci lève l'inhibition de $\mathrm{L} 1$, dont l'émission ne tarde pas à augmenter rapidement. Ces comportements de basculement symétrique ont effectivement été observés à l'aide de photodiodes rapides, mettant en évidence des transitions dont le temps de montée est inférieur à la nanoseconde [4].

Ces résultats montrent d'une part, que le couplage incohérent entre deux lasers à grand gain donne effectivement lieu à une bistabilité optique d'émission, et d'autre part qu'on peut induire optiquement des basculements rapides dans ce type de bistable.

Il est clair, que ce principe de fonctionnement peut être adapté aux lasers à semi-conducteurs, qui présentent eux aussi des milieux actifs de grand gain. C'est bien entendu pour ces lasers que les perspectives d'applications sont les plus intéressantes, en raison de leur compacité, leur possibilité de fonctionnement en régime continu, leur excellent rendement énergétique, et leur compatibilité technologique avec d'autres dispositifs optoélectroniques. On peut remarquer que les cavités utilisées pour les lasers à colorants de l'expérience décrite ci-dessus sont semblables aux cavités des lasers à semi-conducteurs puisque dans les deux cas les miroirs des résonateurs sont constitués par les parois parallèles limitant le milieu actif. On pourrait donc en principe transposer directement la configuration géométrique décrite plus haut au cas de deux lasers à semi-conducteurs. En fait cette transposition soulève une difficulté majeure, due à ce que, dans la plupart des lasers fonctionnant en régime continu, le milieu actif a une structure de guide d'onde à saut d'indice. Le découplage angulaire ne peut donc être réalisé aussi aisément que dans le cas précédent, et il est préférable d'utiliser, avec les lasers à semi-conducteurs, les deux autres types de découplage. En particulier le découplage en fréquence est assez attrayant car il doit permettre une bistabilité de longueur d'onde, permettant en particulier de transposer la longueur d'onde d'un faisceau lumineux porteur d'information. La figure 5 donne le schéma de principe d'un montage en cours d'expérimentation dans notre laboratoire, et devant permettre l'observation de la bistabilité en longueur d'onde avec des lasers à semiconducteurs. Dans ce montage les diodes-lasers sont munies de couches anti-reflets de façon à pouvoir les utiliser en amplificateurs optiques. Chacune des diodes est insérée entre deux objectifs de microscope de façon à collimater les faisceaux en réduisant leur divergence. Les deux cavités résonnantes sont constituées d'une lame semi-réfléchissante commune aux deux sous-cavités et, à chaque extrémité, d'un réseau de diffraction comme nous l'avons déjà décrit sur la figure 3 .

Il est à noter que le dépôt de traitements antireflets de bonne qualité sur les faces clivées des diodeslasers est une étape importante dans la réalisation de ce dispositif bistable. Son intérêt est d'éviter l'apparition de couplages cohérents entre les deux souscavités qui nuiraient à la stabilité de fonctionnement du système. De tels traitements ont été réalisés par dépôt de couches d'oxyde de silicium dont la compo-

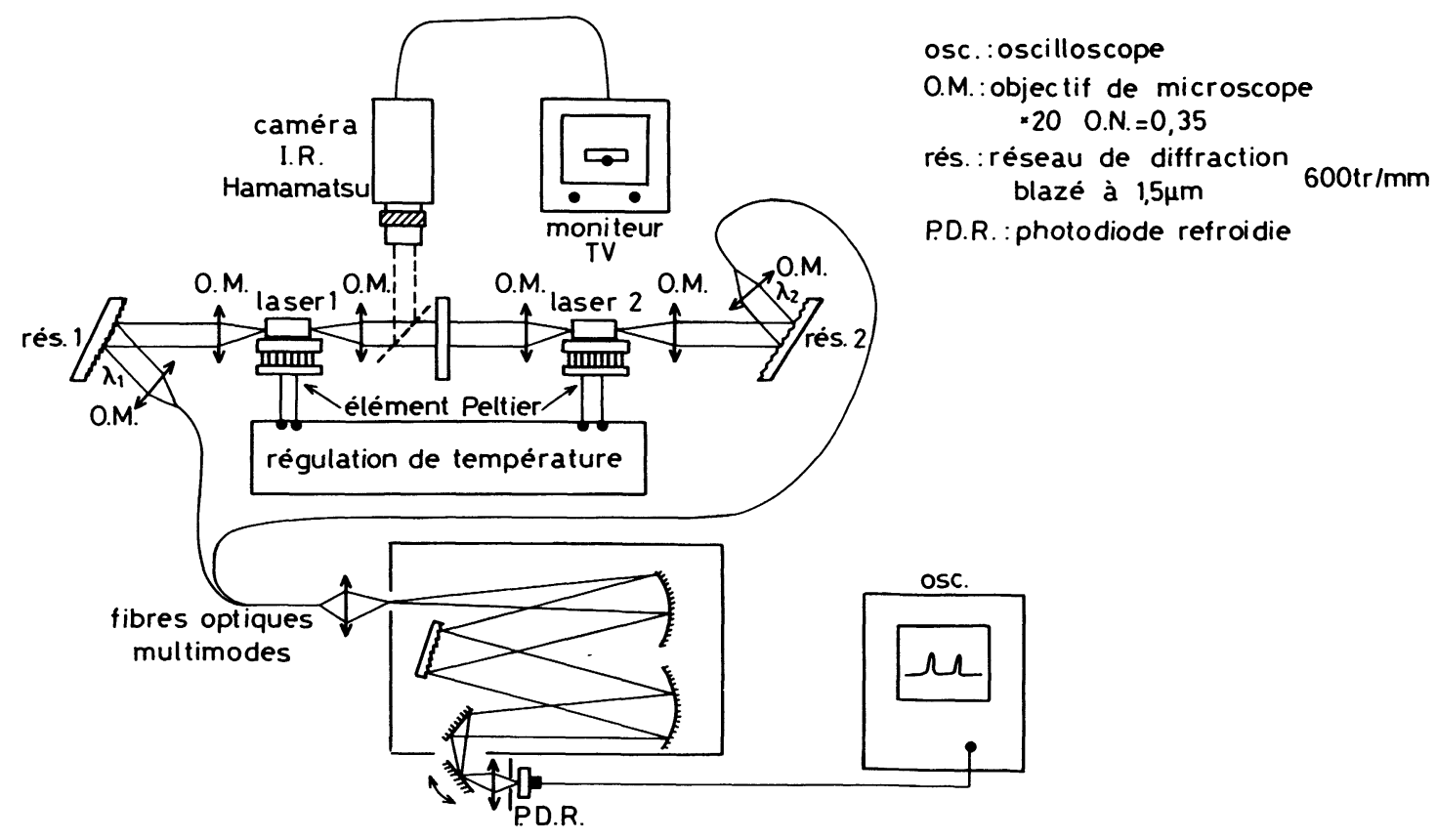

Fig. 5. - Schéma du dispositif bistable à l'étude, utilisant des diodes-lasers [InGaAsP/InP] avec traitement antiréfléchissant, dans des cavités accordables en longueur d'onde par réseau de diffraction.

[Experimental set-up of a bistable device with two cavities, tunable through an external grating and with InGaAsP/InP diode lasers. The facets are anti-reflection coated.] 
sition est ajustée pour obtenir un indice de réfraction voisin de la valeur optimale $n_{\mathrm{opt}}=1,87$, et dont l'épaisseur est de $1720 \AA$ A . Ce dépôt est contrôlé par mesure in situ de la puissance émise par la diodelaser en fonction du courant d'injection.

Une telle diode-laser munie de traitements antireflets a été insérée dans le montage de la figure 5, et le fonctionnement en cavité externe a été observé. La puissance émise a été mesurée en fonction de l'orientation du réseau, mettant en évidence la plage d'accord en longueur d'onde. Sur la figure 6 la puissance émise est représentée en fonction de la longueur d'onde d'émission, et l'on note que le traitement antireflet est de qualité suffisante pour limiter la modulation de l'intensité suivant les modes longitudinaux de la diode-laser (normalement espacés de $10 \AA ̊$ ) à une valeur acceptable.

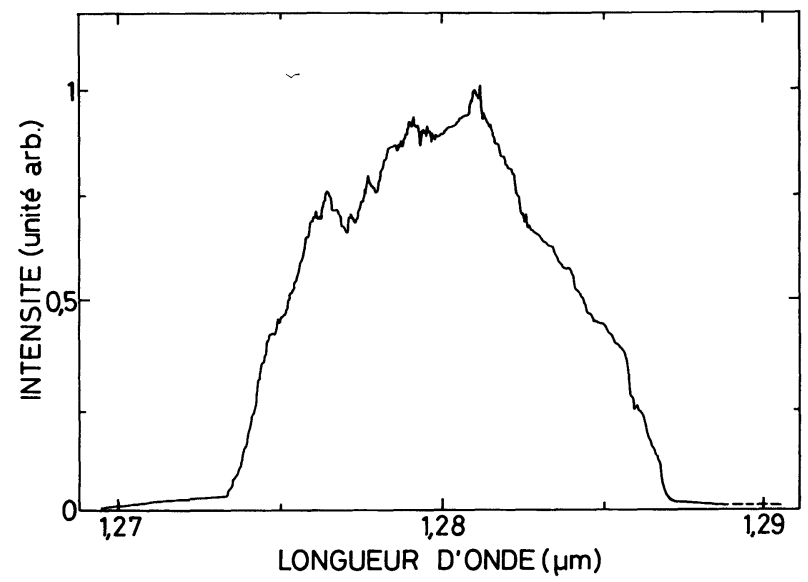

Fig. 6. - Spectre d'accordabilité obtenu pour une cavité unique avec le dispositif de la figure 4 . Le courant d'injection est de $70 \mathrm{~mA}$ pour un seuil de $50 \mathrm{~mA}$. La légère modulation du spectre est due à la réflectivité résiduelle $\left(R \simeq 10^{-2}\right)$.

[Tuning spectrum for a single cavity, obtained with the experimental set-up in figure 4 . The injection current is $70 \mathrm{~mA}$ for a threshold current of $50 \mathrm{~mA}$. The slight modulation of the spectrum is due to the residual reflectivity $\left(R \simeq 10^{-2}\right)$.]

Parallèlement à ce travail expérimental une analyse théorique du fonctionnement de ces dispositifs a été entreprise, de façon à prédire quantitativement le comportement bistable, tant en régime stationnaire qu'en régime transitoire [6].

On considère un système symétrique composé de deux lasers à semi-conducteurs identiques, et dont les interactions sont supposées identiques. Pour les calculs numériques, le cas des lasers à matériau quaternaire InGaAsP/InP est explicitement considéré, d'où la nécessité de tenir compte des processus de recombinaison Auger. Les équations de base sont des équations classiques pour les lasers à semiconducteurs, avec deux termes additionnels pour tenir compte d'une part du couplage non cohérent entre les deux lasers et d'autre part de l'action d'un faisceau extérieur de contrôle.

$$
\begin{gathered}
\frac{\mathrm{d}}{\mathrm{d} t} n_{i}=J_{i}-\frac{n_{i}}{\tau_{\mathrm{sp}}}-A n_{i}^{3}-g\left(n_{i}\right) S_{i}(t)- \\
-\frac{v}{\ell}\left[\exp g\left(n_{i}\right) \frac{\ell}{v}-1\right]\left[\chi S_{j}(t-\theta)+S_{i}^{\mathrm{ex}}(t)\right] \\
\frac{\mathrm{d}}{\mathrm{d} t} S_{i}=\beta \frac{n_{i}}{\tau_{\mathrm{sp}}}+\Gamma g\left(n_{i}\right) S_{i}(t)-\frac{S_{i}(t)}{\tau_{\mathrm{ph}}}
\end{gathered}
$$

avec $i=1$ ou 2 et $j=3-i$ est l'indice complémentaire. Les paramètres physiques régis par ces équations sont :

$$
\begin{aligned}
& S_{i} \quad=\text { densités de photons du laser } \\
& n_{i} \quad=\text { densités de porteurs } \\
& J_{i}=\text { densités de courant normalisées : }[J= \\
& I / e V \text {, où : } \\
& V \quad=\text { Volume du milieu actif; } e: \text { charge de } \\
& \text { l'électron ; } \\
& I \quad=\text { courant d'injection] } \\
& \ell \quad=\text { longueur du milieu actif } \\
& \tau_{\mathrm{sp}} \quad=\text { temps de vie radiatif } \\
& v \quad=\text { vitesse de groupe dans le milieu actif } \\
& A \quad=\text { coefficient Auger } \\
& g\left(n_{i}\right)=\text { coefficient de gain : } g\left(n_{i}\right)=v a\left(n_{i}-n_{0}\right) \\
& n_{0} \quad=\text { densité de porteurs générant la transpa- } \\
& \text { rence } \\
& =\text { facteur de gain } \\
& =\text { facteur de confinement du mode laser } \\
& =\text { facteur d'émission spontanée } \\
& \tau_{\mathrm{ph}} \quad=\text { durée de vie globale du photon incluant } \\
& \text { pertes réflectives et pertes intrinsèques }
\end{aligned}
$$

Le dernier terme de l'équation (2) traduit le taux de réduction de la population sous l'effet de l'interaction avec les faisceaux externes incidents sur le milieu actif du laser $i$ considéré. L'un de ces faisceaux provient de l'autre laser (laser $j$ ) et le coefficient de couplage mutuel $\chi$ est défini comme la fraction de la densité de photons $S_{j}$ de ce laser s'étant propagée jusqu'à l'entrée du milieu actif du laser $i$. La distance entre milieux actifs introduit un retard de propagation $\theta$ sur ce terme d'interaction. L'autre faisceau $\left(S_{i}^{\text {ex }}\right)$ est extérieur au dispositif bistable, et constitue une voie de contrôle optique, par exemple en provenance d'un autre dispositif bistable du même type.

Considérons maintenant le régime stationnaire, obtenu en annulant le second membre des équations (2) et (3). Dans chaque laser, la densité de porteurs est reliée par l'équation (3), à la densité de photons, soit :

$$
n_{i}=\frac{S_{i}}{\tau_{\mathrm{ph}}^{\prime}\left(\beta \tau_{\mathrm{sp}}^{-1}+\Gamma \eta S_{i}\right)}
$$

$$
\eta=v a=\partial\left[g\left(n_{i}\right)\right] / \partial n_{i}
$$


et

$$
\tau_{\mathrm{ph}}^{\prime}=\left(\tau_{\mathrm{ph}}^{-1}+\Gamma \eta n_{0}\right)^{-1} .
$$

De même l'équation (2) fournit une relation entre la densité de photons $S_{i}$, et la densité de photons $S_{j}$ de l'autre laser.

$$
S_{j}=\frac{1}{\chi}\left[\frac{J_{i}-n_{i} \tau_{\mathrm{sp}}^{-1}-A n_{i}^{3}-g\left(n_{i}\right) S_{i}}{(v / \ell)\left(\exp \left[g\left(n_{i}\right) \ell / v\right]-1\right)}-S_{i}^{\mathrm{ex}}\right]
$$

et l'insertion de l'équation (4) dans l'équation (5) permet d'obtenir $S_{2}$ en fonction de $S_{1}$, et réciproquement $S_{1}$ en fonction de $S_{2}$. Comme indiqué schématiquement sur la figure 2 les états stationnaires du système sont obtenus par le calcul des points d'intersection des courbes correspondantes. D'une manière générale la bistabilité se manifeste lorsque les courbes ont trois points d'intersection, le point intermédiaire correspondant à un équilibre instable. On peut alors calculer des courbes d'hystérésis décrivant la puissance de sortie de chaque laser en fonction d'un des paramètres extérieurs au système: $J_{1}$, $J_{2}, S_{1}^{\text {ex }}$ ou $S_{2}^{\text {ex }}$. Sur la figure 7 sont reportées les courbes calculées en considérant la puissance $P_{2}^{\text {ex }}$ comme paramètre de contrôle. En faisant varier le coefficient de couplage mutuel $\chi$, les courants d'injection $J_{1}, J_{2}$ de chaque laser, on peut contrôler la valeur des seuils " haut » et «bas » de basculement. On remarquera que les courants $J_{1}$ et $J_{2}$ choisis pour les courbes de la figure 7 ne sont pas égaux, pour une raison qui sera commentée dans la section 4.

Le régime transitoire peut être calculé à partir des équations (2) et (3), en simulant numériquement une variation plus ou moins rapide d'un des paramè-
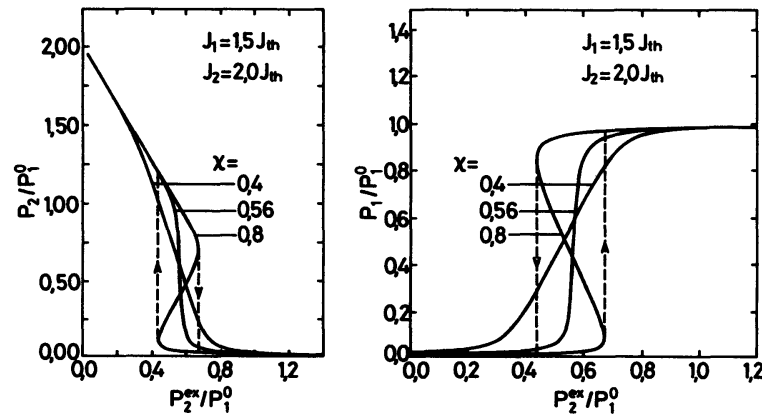

Fig. 7. - Caractéristiques calculées du fonctionnement du dispositif ; puissance de sortie normalisée en fonction de la puissance $P_{2}^{\text {ex }}$ de la commande optique : (a) pour le laser L2 ; (b) pour le laser L1. Ces courbes sont obtenues pour des couplages caractérisant les différents régimes de fonctionnement. Le choix particulier $J_{2}=4 / 3 J_{1}$ permet de décrire la boucle d'hystérésis dans sa totalité.

[Calculated operating characteristics of the device. Normalized output powers versus external beam power $P_{2}^{\text {ex }}$ for (a) laser L2 ; (b) laser L1. These curves are derived for coupling strengths typical of the various operating regimes. The choice $J_{2}=4 / 3 J_{1}$ allows to describe the whole hysteresis loop.] tres extérieurs au système. On dénombre essentiellement trois paramètres temporels gouvernant l'évolution du dispositif : le délai de propagation $\theta$ entre les deux milieux actifs, la durée de vie $\tau_{\mathrm{ph}}$ des photons dans les cavités-lasers et le temps de vie des porteurs dans le milieu actif. Avec les paramètres typiques des lasers à semi-conducteurs (durée de vie radiative $3 \mathrm{~ns}, \tau_{\mathrm{ph}}=16 \mathrm{ps}$ ) et un délai de propagation $\theta$ réduit au minimum, on calcule par simulation numérique [6] un temps de basculement de l'ordre de $100 \mathrm{ps,}$ accompagné d'oscillations de relaxation dont l'amplitude dépend de la géométrie du milieu actif [7] à travers le facteur d'émission spontanée $\beta$. On voit donc que ce type de bistable se prête à la commutation rapide. Les simulations numériques ont également permis d'estimer l'énergie dissipée dans le dispositif au cours d'une commutation : de 1 à $10 \mathrm{pJ}$ suivant la valeur du courant de seuil des lasers utilisés.

\section{Caractéristiques fonctionnelles.}

Le dispositif bistable décrit dans cet article présente des caractéristiques fonctionnelles particulières qu'il est intéressant de comparer à celles que présentent d'autres classes de bistables optiques.

Tout d'abord il s'agit d'un dispositif émetteur de lumière, qui ne nécessite pas, comme les bistables de transmission, la présence d'un faisceau de lumière cohérente destiné à maintenir le système près du seuil de basculement. Par l'emploi de lasers à semiconducteurs la puissance fournie au système peut être purement électrique, et le dispositif bistable peut se maintenir dans un état ou dans l'autre en l'absence de tout signal optique d'entrée : des impulsions optiques (signaux d'entrée) d'une puissance de l'ordre d'une fraction de celle émise par un laser non perturbé c'est-à-dire quelques $\mathrm{mW}$, sont requises uniquement au moment de la commutation. De plus le milieu non linéaire étant intrinsèquement amplificateur, les signaux d'entrée peuvent être d'intensité plus faible que les signaux de sortie, ce qui facilite les mises en cascade de plusieurs de ces dispositifs.

Une deuxième caractéristique importante est la possibilité de réaliser un contrôle électrique du seuil de basculement. Pour l'illustrer, partons de la situation décrite sur la figure 7. Les courbes calculées correspondent à une assez forte dissymétrie dans les courants d'injection $\left(J_{2} / J_{1}=4 / 3\right)$; ainsi même lorsque le couplage ést suffisamment élevé pour que la zone d'hystérésis soit large, le système se trouve naturellement dans l'un des deux états en l'absence de tout signal optique à l'entrée. Si le rapport $J_{2} / J_{1}$ se rapproche de l'unité, la boucle d'hystérésis se rapproche de l'origine, et donc la valeur du seuil de basculement diminue, augmentant ainsi la sensibilité aux faibles signaux d'entrée (Fig. 8). Enfin lorsque les deux courants d'injection sont identiques, 


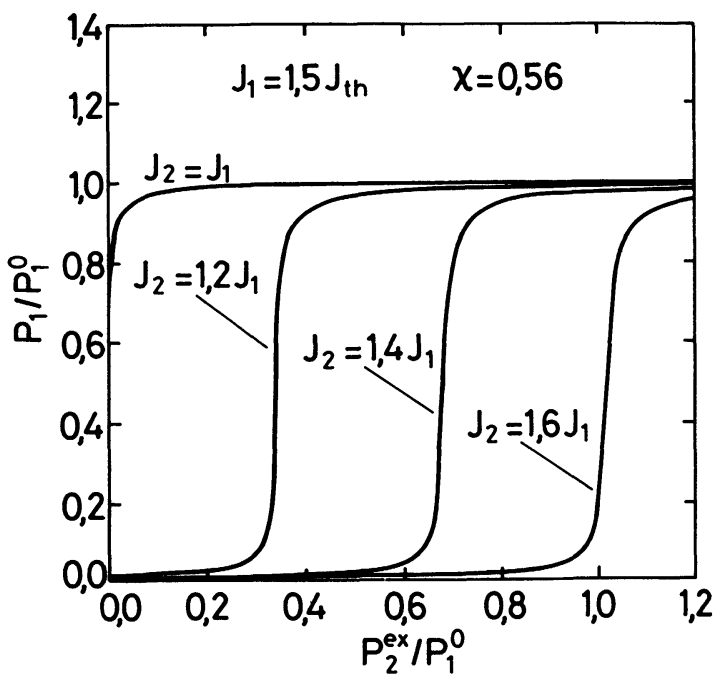

Fig. 8. - Evolution de la caractéristique de fonctionnement de la figure $6 \mathrm{a}$ pour différents rapports des courants d'injection $J_{2} / J_{1}$. La variation de ce rapport permet un ajustement de la position du seuil, modifiant ainsi la sensibilité à un signal optique d'entrée.

[Evolution of the operating characteristic in figure 6a for various injection current ratios $J_{2} / J_{1}$. Varying this ratio allows the adjustment of the threshold position in order to produce the desired sensitivity to optical input.]

seule une demi-boucle d'hystérésis peut être décrite, car sur le diagramme de la figure 7 on a supposé que le signal optique d'entrée n'agit que sur le laser 2.

Remarquons que la possibilité de contrôler électriquement le seuil de basculement, outre la souplesse qu'elle procure pour les fonctions opto-optiques, rend possible également une commutation par voie purement électrique, ce qui permet éventuellement de l'utiliser pour les conversions de signaux logiques électriques en signaux logiques optiques dont la longueur d'onde et l'intensité sont compatibles avec la commande optique de dispositifs semblables.

Enfin une troisième caractéristique intéressante de ce type de dispositif bistable est qu'il comporte, du point de vue optique, deux entrées symétriques et deux sorties symétriques. Pour les sorties cela veut dire en termes de variables logiques que le système génère simultanément un niveau logique et son complémentaire. De même, la nature symétrique des entrées implique que l'on obtient en général le même effet en augmentant l'intensité sur une entrée, ou en la diminuant sur l'autre. Ainsi, dans le cas signalé plus haut où seule une demi-boucle d'hystéré- sis peut être décrite par action sur le laser 2, l'autre moitié de la boucle peut être décrite par une action sur le laser 1 . Cela veut dire que le système peut basculer dans un sens ou dans l'autre, selon l'entrée qui est activée. Ceci constitue une caractéristique précieuse qu'il est rare de rencontrer dans les autres bistables optiques. Dans le cas des bistables de transmission, par exemple, si le système est maintenu dans la zone à hystérésis par un faisceau continu, l'action d'une impulsion lumineuse additionnelle ne peut sauf exception [8], commuter le bistable que dans un sens, celui d'une augmentation de la transmission. Pour induire le basculement dans l'autre sens il faut agir par une diminution de la puissance du faisceau continu de maintien, ce qui complique singulièrement l'utilisation éventuelle de ce type de bistables, par ailleurs fort attrayants par leur simplicité. Le même problème se pose avec les lasers bistables comportant un absorbant saturable intracavité [9], avec lesquels il n'est pas possible de réaliser la fonction d'inversion, ni les fonctions dérivées (NOR et NAND).

\section{Conclusion.}

Les dispositifs qui ont été décrits ici forment une catégorie de bistables optiques dont la structure, le principe et les caractéristiques fonctionnelles les distinguent de bien d'autres bistables optiques. La non-linéarité optique utilisée étant la saturation du gain d'un milieu amplificateur laser, le système est intrinsèquement émetteur de lumière, et donc susceptible de régénérer les signaux optiques. Les possibilités de réalisation avec des lasers à semiconducteurs les rendent aptes d'une part à de bons rendements énergétiques, un faible encombrement, etc. et d'autre part à des traitements logiques adressés électroniquement ou optiquement suivant les besoins. Enfin, la nature symétrique de leur structure est bien adaptée à une utilisation pour traiter des signaux optiques en codage binaire, de même que les bascules symétriques sont très répandues dans les circuits électroniques numériques.

\section{Remerciements.}

Nous remercions vivement J. C. Bouley, H. Nakajima et $\mathrm{J}$. Landreau pour la fourniture de lasers à semi-conducteurs et la réalisation des traitements antiréfléchissants. 


\section{Bibliographie}

[1] Voir par exemple : Proc. IEEE 72 (1984) - special issue on optical computing.

[2] Pauliat, G., Heniau, J. P., Delboulbé, A., RooSEN, G. et HuignaRd, J. P., Josa B 3, 306 (1986) - Goodman, J. W. et al. pp. 850-866 de [1] ci-dessus.

[3] GibBS, H. M., Optical bistability : controlling light with light. (Academic press, N.Y.) 1985.

[4] Oudar, J. L. et KuszelewiCz, R., Appl. Phys. Lett. 45 (1984) 831.

[5] Agrawal, G. P. et Dutta, N. K., J. Appl. Phys. 56 (1984) 664.
[6] Kuszelewicz, R. et Oudar, J. L., soumis à IEEE J. Quantum Electron.

[7] Boers, P. M. et VlaARdingerbroek, M. T., Electron. Lett. 11 (1975) 206.

[8] Tarng, S. S., Tai, K., Jewell, J. L., Gibbs, H. M., Gossard, A. C., Mc Call, S. L., Passner, A., Venkatesan, T. N. C. et WiegmanN, W., Appl. Phys. Lett. 40 (1982) 205.

[9] Nathan, M. I., Marinace, J. C., Rutz, R. F., Michel, A. E. et Lasher, G. J., J. Appl. Phys. 36 (1965) 473. 